\title{
Recent Progress in non-Fermi Liquid Theory in Two Dimensions
}

Low-energy effective theory of Fermi surface coupled with $U(1)$ gauge field in $2+1$ dimensions

Author: Sung-Sik Lee,

Phys. Rev. B 80, 165102 (2009)

Quantum phase transition of metals in two spatial dimensions. I. Ising-nematic order Authors: Max Metlitski and Subir Sachdev, Phys. Rev. B 82, 075127 (2010).

A Controlled Expansion for Certain non-Fermi Liquid Metals

Authors: David Mross, John McGreevy, Hong Liu and T. Senthil,

Phys. Rev. B 82, 045121 (2010)

\section{Recommended and a Commentary by Patrick Lee, Massachusetts Institute of Technology}

Largely inspired by the unusual phenomenology observed in high temperature superconductors, the search for models which exhibit violation of Landau's Fermi liquid description has been a focus of research on strong correlation physics in the past two decades. Inconveniently, the superconducting state intervenes, making it difficult to access the purported exotic ground states and the applicability of these ideas to Hi $T_{c}$ remains under debate. On the other hand, an outgrowth of this research is the revival of the notion of RVB and quantum spin liquid and the associated emergent particles such as spinons and gauge fields. The recent discovery of several candidate spin liquid materials which appear to be gapless has stimulated interest in the models involving fermionic spinons coupled to $U(1)$ gauge fields.[1] 
(Bosonic spinons are typically gapped.) A typical action of interest is $S=S_{f}+S_{i n t}+S_{a}+S_{d}$.

$$
\begin{aligned}
S_{f} & =\sum_{k, \alpha} f_{k, \alpha}^{\dagger}\left(-i \omega+\varepsilon_{\boldsymbol{k}}-\mu\right) f_{k, \alpha} \\
S_{i n t} & =\sum_{\boldsymbol{q}, \omega} a(\boldsymbol{q}, \omega) j(\boldsymbol{q}, \omega) \\
S_{a} & =\sum_{\boldsymbol{q}, \omega} \frac{q^{2}}{e^{2}}|a(\boldsymbol{q}, \omega)|^{2}
\end{aligned}
$$

where $S_{d}$ is the diamagnetic term. Here $f_{k, \alpha}$ is the fermionic spinon operator with $k=(\boldsymbol{k}, \omega)$, $\alpha$ is the spin label which may be expanded to $N$ flavors $\alpha=1 \cdots N, a(\boldsymbol{q}, \omega)$ is the transverse component of a $U(1)$ gauge field and $j(\boldsymbol{q}, \omega)$ is the transverse fermion current density. The reader may recognize this as the familiar $\boldsymbol{A} \cdot \boldsymbol{j}$ coupling of electrons to electromagnetic fields. Indeed, the only differences are that in spin liquids the gauge field is $2 \mathrm{D}$ and has been introduced to enforce constraints. Therefore, $e^{2}$ is initially infinite, i.e., the Maxwell term Eq.(3) is absent. However, by integrating out high energy fermions, Eq.(3) will be generated with $e^{2}$ of order unity. We focus on the transverse component because unlike longitudinal density fluctuations, transverse currents are not screened and lead to singular small $q$ and $\omega$ behavior. This fact was recognized long ago by Holstein, Norton, and Pincus,[2] who pointed out that even in our 3 dimensional world, coupling of electrons to transverse gauge fluctuations leads to a specific heat which goes as $T \ln T$, in violation of Landau's Fermi liquid theory. Due to the small size of the fine structure constant and $v_{F} / c$, this effect is very small and has never been observed to my knowledge. Reizer extended this to $2 \mathrm{D}$ and showed that the specific heat goes as $T^{2 / 3}$ while the fermion self energy goes as $\omega^{2 / 3}$. [3, 4] This is a clear signature of non-Fermi liquid behavior, because the quasiparticle pole is replaced by a power law singularity. The origin of this singularity is that Landau damping converts Eq.(3) to an effective action of the form

$$
S_{a}^{\prime}=\sum_{\boldsymbol{q}, \omega}\left(\frac{i \omega}{v_{F} q} N(0)+\chi q^{2}\right)|a(\boldsymbol{q}, \omega)|^{2}
$$

where $N(0)$ is the fermion density of states and $\chi$ is the Landau diamagnetic susceptibility. The gauge field becomes overdamped, with $\omega$ scaling as $q^{3}$. The abundance of soft gauge fields relaxes the fermions rapidly, leading to a violation of Landau's condition that the quasiparticle relaxation rate must be smaller than its energy.

Equations (1-3) also describe the nematic quantum critical point in metals, where the Fermi surface spontaneously distort to beak the lattice symmetry.[5] Signs of this nematic 
transition have been seen in $\mathrm{Sr}_{3} \mathrm{Ru}_{2} \mathrm{O}_{7}$ [6] and via the Nernst signal in $\mathrm{YBa}_{2} \mathrm{Cu}_{3} \mathrm{O}_{7}$. [7] In this case $a$ is a real field called the Ising nematic order parameter and $j$ in Eq.(2) is replaced by a suitable fermion bilinear operator. This is also referred to as the Pomeranchuk instability of the Fermi surface.

The original work on Eqs.(1-3) was based on the RPA.[2-4] Over the years a great deal of physical insights have been gained. Notably, Altshuler, Ioffe and Millis [8] and Polchinski [9] formulated a $\frac{1}{N}$ expansion and emphasize that since the transfer of small $q$ and $\omega$ boson is important, in the large $N$ limit a given Fermi momentum $\boldsymbol{k}_{0}$ is scattered to a nearby $\boldsymbol{k}$ point by a $\boldsymbol{q}$ vector which is almost tangential to the local Fermi surface. It was shown that the gauge field propagation is well described by Eq.(4) and the common belief was that a $\frac{1}{N}$ expansion is possible.

The recent reexamination on this problem began with the paper by Sung-Sik Lee. Building on the insight of [8] and [9], Lee formulated a low energy effective action involving small patches around a given $\boldsymbol{k}$ point. Two kinds of processes are important. The first involves scattering between $\boldsymbol{k}$ vectors which are nearly parallel to each other (the one patch model) and the second involves small angle scattering between $\boldsymbol{k}$ points on patches with parallel but opposite normals (called the two patch model). Lee then carried out a careful analysis of the Feynman diagrams. He showed that even for the one patch model, the $\frac{1}{N}$ expansion is not convergent, i.e., an infinite summation of Feynman diagrams is needed at every order in $\frac{1}{N}$. He was able to organize these diagrams as planar diagrams characterized by the genus number of the two dimension surface on which the Feynman diagrams are drawn, reminiscent of the observation by t'Hooft for large $N$ QCD. Since the problem remains a strongly coupled one, there is no way to compute these diagrams in a controlled manner. The hope for a $\frac{1}{N}$ treatment of Eqs.(1-3) is dashed.

Metlitski and Sachdev followed up on Lee's work and formulated a scaling theory of the two patch model. They carried out a loop expansion and discovered a singular momentum dependence of the fermion self energy at three loop order. However, with the two patch model the difficulty of the $\frac{1}{N}$ expansion discovered by Lee is further amplified and a controlled calculation is not possible.

What went wrong with the $\frac{1}{N}$ expansion? The traditional view is that the gauge field propagation is of order $\frac{1}{N}$ because it is a string of fermion bubbles. If the fermion Green's function is of order 1 , the expectation was that a loop expansion will provide a controlled 
$\frac{1}{N}$ expansion. What Sung-Sik Lee noted is that while the fermion self energy is of order $\frac{1}{N}$, there are higher order loop diagrams which are divergent and need to be regularized by the self energy itself. The fact that the self energy is of order $\frac{1}{N}$ comes back to haunt us and leads to a large contribution of these diagrams, invalidating the naive expectation. While Lee's theory is highly elegant, it also created a deep sense of pessimism. It looks like we do not have a controlled example of a non-Fermi liquid theory in 2D after all.

Fortunately, this pessimism is short-lived and Mross et al. came to the rescue. They found that the difficulties pointed out by Sung-Sik Lee can be bypassed formally by introducing a double expansion. To be more precise, they introduced an extra parameter $z_{b}$ in the model by replacing Eq.(3) by

$$
S_{a}^{\prime}=\sum_{\boldsymbol{k}, \omega} \frac{|\boldsymbol{k}|^{z_{b-1}}}{e^{2}}|a(\boldsymbol{k}, \omega)|^{2} .
$$

The original problem corresponds to $z_{b}=3$. The case $z_{b}=2$ arises in the composite fermion description of the half-filled Landau level in the presence of $\frac{1}{r}$ Coulomb interaction.[10] It is known that the self energy $\Sigma$ goes as $\omega \ln |\omega|$ and it is natural to expand around $z_{b}=2$. The double expansion they propose is $N \rightarrow \infty, \varepsilon=z_{b}-2 \rightarrow 0$ such that $\varepsilon N$ is finite. The reason this works is the following. The fermion self energy now takes the form

$$
\Sigma=-i \frac{1}{\lambda N} \operatorname{sign}(\omega)|\omega|^{\frac{2}{z_{b}}}
$$

where

$$
\lambda=4 \pi \sin \left(\frac{2 \pi}{z_{b}}\right) \gamma^{\frac{z_{b}-2}{z_{b}}}
$$

with $\gamma=\frac{1}{4 \pi}$. Note that $\lambda N \propto \varepsilon N$ and goes to a constant in the limit chosen above. The self energy then provides a finite cutoff for the divergent diagrams Lee was concerned with, and a well defined loop expansion is restored. It should be noted that an $\varepsilon$ expansion for any fixed $N$ was formulated by Nayak and Wilczek earlier and computed to first order in $\varepsilon$.[11] Where they overlap the two methods agree, but the double expansion has the advantage that higher order loop expansion is more easily evaluated. Indeed, taking advantage of the hard work already done by Metlitski and Sachdev, Mross et al. evaluated the anomalous dimension for the fermion $\eta_{f}$ to order $\frac{1}{N^{2}}$. The result turns out to be different for the nematic critical point and the gauge field problem. In the former case $\eta_{f}$ has a positive correction of order $\frac{1}{N^{2}}$ while in the latter case, $\eta_{f}$ is negative and less dominant. Mross et al. also computed the $2 k_{f}$ response, confirming the enhancement predicted by Altshuler et al. [8] for the gauge field problem. 
While there is no guarantee that the physical problem $N=2, \varepsilon=1$ is smoothly connected to the large $N$ small $\varepsilon$ limit, the recent reexamination has greatly deepened our understanding of this important class of problem. We can rest assured that a well formulated example of non-Fermi liquid state exists.

[1] See P.A. Lee, Science 321, 1306 (2008) for additional references.

[2] T. Holstein, R. Norton and P. Pincus, Phys. Rev. B 8, 2649 (1973).

[3] M. Reizer, Phys. Rev. B 40, 11571 (1989).

[4] P.A. Lee and N. Nagaosa, Phys. Rev. B 46, 5621 (1992).

[5] See S. Kivelson et al., Rev. Mod. Phys. 75, 1201 (2003) and the Metlitski-Sachdev paper for additional references.

[6] R. Borzi et al., Science 315, 214 (2007).

[7] R. Daou et al., Nature 463, 519 (2010).

[8] B. Altshuler, L. Ioffe and A. Millis, Phys. Rev. B 50, 14048 (1994).

[9] J. Polchinski, Nucl. Phys. B 422, 617 (1994).

[10] B. Halperin, P. Lee and N. Read, Phys. Rev. 7312 (1993).

[11] C. Nayak and F. Wilczek, Nucl. Phys. B 430, 534 (1994). 